Paper

\title{
Modeling of Fluid Dynamics and Thermo-Chemical System in Halogen Lamp Operation
}

\section{Makoto BESSHO* and Masashi OHKAWA**}

\author{
*Toshiba Lighting \& Technology Corp. \\ **Niigata University
}

Paper originally published in Japanese in J. IEIJ, Vol. 93, No.11, 2009

\begin{abstract}
One of the key elements in improving lamp efficiency is understanding and controlling the halogen transport process. Therefore, it is worthwhile to build a model that depicts this process and can be applied to the study of various types of lamps. We examined the halogen transport process in a lamp by using a hybrid simulation model based on fluid dynamics and chemical equilibrium.
\end{abstract}

KEYWORDS: halogen lamp, equilibrium, halogen cycle, CFD, free energy, tungsten

\section{Introduction}

Since first being put to practical use in 1959 by Dr. Zubler of General Electric, the halogen lamp has evolved as an energy-efficient light source. A halogen lamp emits light by incandescent emission, which is the same light emitting principle as that of an incandescent bulb, and the application of the so-called halogen cycle reaction, in which tungsten that has adhered to the bulb combines with halogen and vaporizes, enabling an excellent luminous flux maintenance factor in the range of $90 \%$ to $100 \%$ to be obtained throughout the life of the bulb. Even today, with the popularization of other highly efficient light sources such as fluorescent lamps and high intensity discharge lamps, the halogen lamp remains highly regarded due to its high efficiency of light utilization owing to a small lamp size, excellent light quality with a color rendering index value of nearly 100 , ease at which light can be dimmed, and so on.

Until about the mid-1980s, in published reports concerning heat transport, substance transport, chemical reactions and the like occurring inside the bulb of a halogen lamp, the change in partial pressure of formed products corresponding to temperature had been investigated by computing the chemical equilibrium of the reacting system that includes halogen ${ }^{1 /-8}$. The equilibrium calculations were carried out using an equilibrium constant, and therefore the substances that could be handled by this method were limited to gases. Beginning in the mid-1980s, the handling of solid-phase substances was made possible with the practical application of a calculation method that minimizes the Gibbs free energy ${ }^{9)}$ 16). In published reports from this period, the entire space for which calculations are to be carried out was assumed to be a thermodynamically homogenous system, and the type of composition obtained in the equilibrium state was calculated with the filament temperature as a variable. Comparisons of the calculated results with experimental results yielded a variety of useful knowledge ${ }^{17-19)}$. The largest difference between the calculated and actual phenomena was that the assumption of a homogeneous system for the entire area of the equilibrium state calculation differed from the actual conditions inside an actual bulb. Inside an actual bulb, the composition is not homogeneous and will vary depending on location, and after lighting, the composition will change over time. In other word, the phenomena of actually occurring chemical changes could not be reproduced as a direct model.

Upon entering the 1990s, as a result of advances in computer technology and analysis codes, thermo fluid field simulations were reported ${ }^{20)} \cdot 21$. Heat transport phenomena inside a bulb could be now be handled numerically, and the meaning and true state of the Langmuir sheath ${ }^{22}$ proposed by Langmuir could be verified by simulations. Namely, on the inner side of the Langmuir sheath, the primary form of the transport phenomenon is diffusion, while on the outer side, the effect of convection is larger, and additionally, the behavior also depends on the temperature distribution and pressure. Also in the 1990s, a halogen lamp was developed that exhibits no significant deterioration in performance characteristics despite having been formed with a low filling pressure, and the consideration of intra tubular phenomena provided invaluable insights in the development of this lamp.

However, even in these published reports, chemical reactions and thermo fluid fields were not analyzed 
simultaneously. At present, several codes for analyzing thermo fluid fields have been used in practical applications, but since these codes are not supplied with a general thermodynamic database, methods that combine thermo fluid field analysis and chemical reaction analysis have not yet become commonplace, and a detailed report of an analysis method was not found.

In this study, three analytic software programs (thermo fluid field analysis, chemical equilibrium analysis, and the interfaces thereof) are combined to simulate actual intra tubular phenomena in association with the behavior of thermo fluids and chemical equilibrium. The results obtained are discussed herein.

\section{Theory}

\subsection{Thermo-fluid analysis}

The equations governing non-reactive thermo fluid fields are the independent equations (1) to (3) shown below ${ }^{23244)}$. In the case of a reactive thermo fluid field, equation (4) is also added23)24.

(a) Mass conservation equation

The law of conservation of mass of the entire system is expressed as an equation of continuity and can be written as follows.

$$
\frac{D \rho}{D t}+\rho(\nabla \cdot v)=0
$$

Here, $v$ is the flow velocity and $\rho$ is the density. Also, $D D t$ is the actual differential operator.

$$
\frac{D}{D t} \equiv \frac{\partial}{\partial t}+\frac{\partial}{\partial x}+\frac{\partial}{\partial y}+\frac{\partial}{\partial z}
$$

\section{(b) Motion equation}

The equation of motion can be derived from an equation that equates the sum of the momentum in and out of a micro volume element and the force applied externally to the change in momentum per unit time in the volume element. In the case of a Newtonian fluid, this equation can be written as follows.

$$
\frac{\partial}{\partial t}(\rho v)=-\nabla \cdot(\rho v v)-\nabla p+\nabla \cdot \tau+\rho f
$$

Here, $r$ is the viscous stress tensor, $f$ is the external force (body force) and $\boldsymbol{p}$ is pressure. Also, $\rho \boldsymbol{v} \boldsymbol{v}$ is the momentum-velocity tensor and can be written as follows, where the component of $v$ in each direction is $v_{x}, v_{y}$ and $v_{z}$.

$$
\rho \boldsymbol{V} \boldsymbol{V}=\rho\left(\begin{array}{lll}
v_{x} v_{x} & v_{x} v_{y} & v_{x} v_{z} \\
v_{y} v_{x} & v_{y} v_{y} & v_{y} v_{z} \\
v_{z} v_{x} & v_{z} v_{y} & v_{z} v_{z}
\end{array}\right)
$$

Equation (2) is called the Navier-Stokes equation, where the 1st term on the right-hand side is the momentum flowing into the micro volume element, and the 2nd and 3 rd terms express the force acting on a micro volume element as a static pressure component acting in the direction perpendicular to the surface and a component acting parallel to the surface, respectively. The static pressure component is expressed as pressure and the parallel component is expressed as the viscous stress tensor. The 4 th term on the right-hand side is the amount of work due to an external force.

The viscous stress tensor $\tau$ is given by the following equation.

$$
\boldsymbol{\tau}=\left\{\left(\mu^{\prime}-\frac{2}{3} \mu\right)(\nabla \cdot v) /\right\}+\mu\left\{(\nabla v)+(\nabla v)^{\mathrm{T}}\right\}
$$

Here, $\mu$ denotes the viscosity coefficient, $\mu^{\prime}$ denotes the bulk viscosity, $I$ denotes the unit tensor, and subscript $T$ denotes the transpose matrix. Moreover, $\nabla v$ is the velocity gradient tensor and is defined as in the following equation.

$$
\nabla v=\left(\begin{array}{lll}
\frac{\partial v_{x}}{\partial x} & \frac{\partial v_{y}}{\partial x} & \frac{\partial v_{z}}{\partial x} \\
\frac{\partial v_{x}}{\partial y} & \frac{\partial v_{y}}{\partial y} & \frac{\partial v_{z}}{\partial y} \\
\frac{\partial v_{x}}{\partial z} & \frac{\partial v_{y}}{\partial z} & \frac{\partial v_{z}}{\partial z}
\end{array}\right)
$$

\section{(c) Energy equation}

The energy equation expresses that the energy flowing into a certain volume within a system is equal to the time variation of the total energy contained in this volume. When considering a thermo fluid, the total energy is usually assumed to consist of thermal energy (enthalpy) and kinetic energy. In the case of a single-component system, the energy equation is

$$
\rho \frac{\partial h}{\partial t}=-\nabla \cdot q+\frac{\partial p}{\partial t}+\Phi
$$

where, $\boldsymbol{l}$ is enthalpy, $\boldsymbol{q}$ is heat flux, and $\Phi$ is the viscous dissipation of energy.

In the case of a multi-component system, the enthalpy change that accompanies the flow of each chemical species must also be added. With different chemical species, the specific heat differs even at the same temperature, and therefore the enthalpies will also be different. Accordingly, the energy equation is

$$
\rho \frac{\partial h}{\partial t}=-\nabla \cdot\left(q+\sum_{i} \rho_{i} V_{i} h_{i}\right)+\frac{\partial p}{\partial t}+\Phi
$$

where, $v_{i}$ is the velocity relative to the average flow velocity and $\boldsymbol{h}_{\boldsymbol{i}}$ is the specific enthalpy. Also, $\rho_{i}$ is the mass fraction of the chemical species $i$, and $\sum_{i} \rho_{i}=\rho$ is satisfied. $\sum_{i} \rho_{i} v_{i} h_{i}$ in the 1 st term on the right-hand side of Equation (3) expresses the sum total of enthalpy transported by the chemical species $i$. 
(d) Chemical species conservation equation and mass transfer

With a multi-component system fluid, because the configuration of the chemical species changes temporally and spatially, governing equations are needed for each chemical species. The chemical species conservation equation expresses that the mass of a chemical species flowing into a certain volume in the system does not change before and after a reaction. Since the number of moles does change before and after a reaction, mass is considered rather than the number of moles. In a multicomponent system, the chemical species conservation equation (transport equation) is:

$$
\frac{\partial \rho_{i}}{\partial t}+\nabla \cdot\left(\rho_{i} \mathbf{v}_{i}-D_{i m} \nabla \rho_{i}\right)=w_{i} \quad \cdots \cdots(4)
$$

where, $\boldsymbol{D}_{i m}$ is the molecular diffusion coefficient for the mixture $\boldsymbol{m}$ of the chemical species $\boldsymbol{i}, \boldsymbol{w}_{\boldsymbol{i}}$ is the amount of chemical species $i$ produced per unit time and per unit mass, and $v_{i}$ is the flow velocity of chemical species $i$.

The equations described in paragraphs (a) to (d) are balance equations describing, through changes in the system, the preservation of each conservation quantities, and by solving them, the status of each time series can be known. Here, the composition of each time series is obtained by a chemical equilibrium calculation, and the results are plugged into equations (a) to (d), and thermo fluid analytical calculations are carried out.

\subsection{Chemical equilibrium calculation}

The chemical equilibrium software program used in this study is FactSage ${ }^{\mathrm{TM}}$, and by minimizing the Gibbs free energy, the chemical equilibrium, including the condensed phases, could be calculated.

In the case of a halogen lamp, a liquid phase does not occur among the condensed phases, but in the solid phase, tungsten, tungsten oxide, tungsten halide and the like are formed at areas of low temperature on the inner surface of the bulb or in the periphery of the filament, and therefore the condensed phases must also be included in the calculations. The calculation method is described in the reference literature ${ }^{9 \cdot 13}$, and an overview is presented herein.

The Gibbs free energy of the system, $G$, can be expressed as

$$
G=\sum_{i} x_{i} g_{i}
$$

where $x_{i}$ is the number of moles of the compound and $g_{i}$ is the chemical potential. Here, $g_{i}$ is defined as:

$$
g_{i}=g_{i}^{0}+R T \ln a_{i}
$$

The gaseous phase activity $a_{i}$ matches the partial pressure $p_{i}$ and can be expressed as:

$$
a_{i}=p_{i}=\frac{x_{i}}{X} P
$$

where, $\boldsymbol{X}$ and $\boldsymbol{P}$ are the total number of moles and total pressure, respectively, of the gaseous phase.

However, if equation (5) is separated for the gaseous phase and condensed phases and then summed, in the case of an condensed compound, since $a_{i}=1$, the 2 nd term on the right-hand side of equation (6) becomes 0 , and the equation can be written as:

$$
\frac{G}{R T}=\sum_{i=1}^{m} x_{i}^{\mathrm{g}}\left\{\left(\frac{g^{0}}{R T}\right)_{i}^{g}+\ln P+\ln \left(\frac{x_{i}^{\mathrm{g}}}{X}\right)\right\}+\sum_{i=1}^{s} x_{i}^{\mathrm{c}}\left(\frac{g^{0}}{R T}\right)_{i}^{\mathrm{c}}
$$

$$
\cdots \cdots(7)
$$

where, $R$ is the gas constant, $T$ is the absolute temperature, $\boldsymbol{m}$ is the number of chemical species comprising the gaseous phase, and $s$ is the number of chemical species comprising the condensed phases. Moreover, the superscripts $\mathbf{g}$ and $\mathbf{c}$ express the gaseous phase components and condensed phase components, respectively. Furthermore, the value of $g \%(R T)$ is calculated according to the following equation for each compound.

$$
\frac{g^{0}}{R T}=\frac{1}{R} \frac{G^{0}-H_{298}^{0}}{T}+\frac{\Delta_{f} H_{298}^{0}}{R T}
$$

Here, $\left(G^{0}-H_{298}^{0}\right) / T$ is the free energy function and $\Delta_{f} H_{298}^{0}$ is the enthalpy produced at standard temperature. Moreover, the superscript 0 and subscript 298 express the thermodynamic standard state and standard temperature $\left(25^{\circ} \mathrm{C}=298.15 \mathrm{~K}\right)$, respectively.

On the other hand, the mass balance is:

$$
\sum_{i=1}^{m} a_{i j}^{g} x_{i}^{g}+\sum_{i=1}^{s} a_{i j}^{c} x_{i}^{c}=b_{j} \quad j=1,2, \cdots l \cdots(8)
$$

where $a_{i j}$ is the number of atoms of component $j$ (jth element) among the elements comprising 1 mole of the ith compound, $b_{j}$ is the total number moles of the th component, and $l$ is the total number of constituent elements.

The equilibrium composition is the combination of $x_{i}^{\mathrm{g}}$ and $x_{i}^{\mathrm{c}}$ which, based on conditions that satisfy the depended condition equation (8), minimizes the Gibbs free energy expressed by equation (7). The Lagrangean undetermined multipliers method was used as the minimization method. 


\section{Analytical model}

\subsection{Configuration of analytical model}

\subsubsection{General analytical procedure}

First, the temperature field and convective velocity field inside a bulb are calculated with a $\mathrm{Kr}-\mathrm{N}_{2}$ system filled at $3.04 \times 10^{5} \mathrm{~Pa}$ (3 atm). Here, the $\mathrm{Kr}$ fraction is 0.9 and the remain is $\mathrm{N}_{2}$. The chemical equilibrium is calculated at the temperature and pressure determined for each cell, and the composition of each cell is obtained. This composition will differ from that of adjacent cells, and therefore the diffusion of concentration is considered for each chemical species, whereby the chemical species flows to adjacent cells. This series of calculations is repeated, and when the partial pressure change of the specific compound being monitored is less than $10^{-3} \mathrm{~Pa}$ when compared over a continuous time interval, and the graph of the transition of partial pressure change for all chemical species is visually observed to be stable, a steady state is considered to have been reached, and the calculation is complete.

With the model used in this study, a quantity known as the reaction ratio is defined. If the chemical reaction rate is sufficiently fast, the reaction ratio is $100 \%$, and chemical equilibrium is fully established in each cell at all times. However, verification by measurement has not yet been carried out, and therefore, assuming that the chemical reaction has not been completely established, the amount of chemical species that leads to an equilibrium state as a result of the chemical reaction in each cell is defined as the reaction ratio so as to correspond flexibly to future research results. Both the chemical species that led to equilibrium and the various residue chemical species are transferred by convection and diffusion. As will be described below, because the time required for the partial pressure distribution within the system to stabilize after lighting is at least four orders of magnitude smaller than the $3000 \mathrm{hr}$ service life of the lamp, even assuming a fluctuation in the reaction ratio, there is not believed to be any effect on the partial pressure distribution when stable. Moreover, it is confirmed by visual observation that chemical changes progress moderately throughout the service life of the lamp, and nearly all periods throughout the service life are considered to be stable.

\subsubsection{Hybrid thermo fluid-chemical equilibrium calculations}

First, the boundary conditions for accurately reproducing the temperature distribution of an actual halogen lamp (bulb surface temperature and filament temperature) are determined, and then based on the obtained boundary conditions, the velocity and temperature allocated to each micro cell that subdivides the interior of the bulb are calculated. This calculation is performed using a three-dimensional model. However, performing chemical equilibrium calculations in order to determine the partial pressures of the chemical species contained within each cell will place an extremely large burden on the computer, and it is not yet practical to perform all calculation processes in three dimensions.

Therefore, the chemical equilibrium calculations are performed in two dimensions. The two-dimensional model described herein is actually three-dimensional model that is one pixel thick, and the analysis results for the $y=0$ cross-section of the three-dimensional flow field and temperature field obtained in the previous step are converted into this new two-dimensional model data. However, by eliminating the $y=0$ crosssection and converting the model into a two-dimensional model as above, the flow field does not satisfy the continuity equation. Therefore, an external force (to be described later) is used to correct the field flow so that the continuity equation is satisfied with the two-dimensional model, and based on the newly created thermo fluid field, the chemical equilibrium is calculated.

Next, using the recalculated thermo fluid field, the time variation of the partial pressure of each chemical species is calculated. Equilibrium calculations are carried out for each cell every $0.5 \mathrm{sec}$, and the results are passed to the adjacent cells by diffusion and convection. The specific chemical species of a specific cell is monitored, and when the temporal variance of the calculated results is less than the predetermined threshold value of $10^{-3} \mathrm{~Pa}$ and the graph of the transition of partial pressure change for all chemical species is visually observed to be stable, a steady state is considered to have been reached, and the calculation is complete. Here, the $10^{-3} \mathrm{~Pa}$ does not have any general significance, but was set so that the partial pressures would be less than the SPW minimum value in the equilibrium calculations.

\subsection{Modeling of thermo fluid field}

\subsubsection{Shape of lamp and filament}

Figure 1 shows the appearance of the halogen lamp under consideration. The lamp is a commercial voltage halogen lamp integrated with a mirror, which is widely used for shop lighting and the like. To control the luminous intensity with a small mirror having an outer diameter of $51 \mathrm{~mm}$, and in order to enhance the light utilization efficiency, the coil length is designed to be shorter than the total coil length of the halogen lamp without the mirror. Consequently, the temperature distribution of the bulb is 10 to $50 \mathrm{~K}$ higher than that of a halogen lamp having the same power consumption without a mirror. Furthermore, the coordinate system was designed as shown in Figure 2. The origin is the light emitting end at the top of the bulb. Assuming that lamp is illuminated with base facing upward, the $z$-axis is positive in direction of the sealed part side. 
When modeling the coil, one must consider the extent to which accurate modeling will be pursued. Therefore, in order to consider the necessity of coil leads that connect to both ends of the light emitting part of the filament, the peripheral thermo fluid fields were compared for the cases in which the coil leads are and are not included. Figure 3 shows the results of a comparison of the convection status for the cases in which a coil lead was and was not attached to both ends of the filament. In the case shown in Figure 3(a) in which a coil lead is not attached to one side of the filament, the appearance of the flow velocity vector at the ends of the coil differs greatly depending on whether or not a coil lead is present.

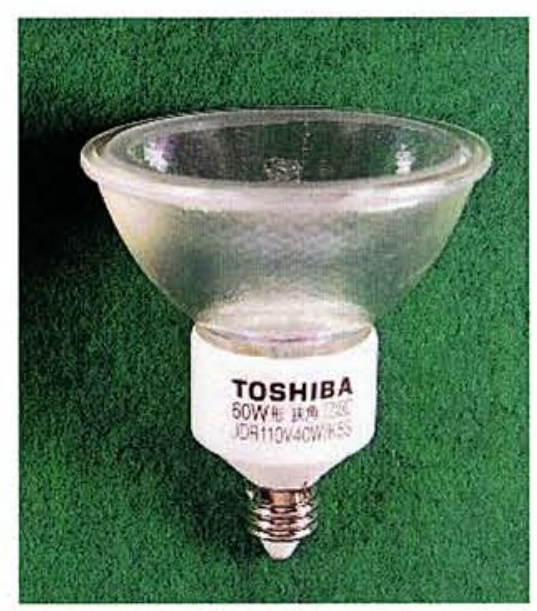

Figure 1 Appearance of the lamp

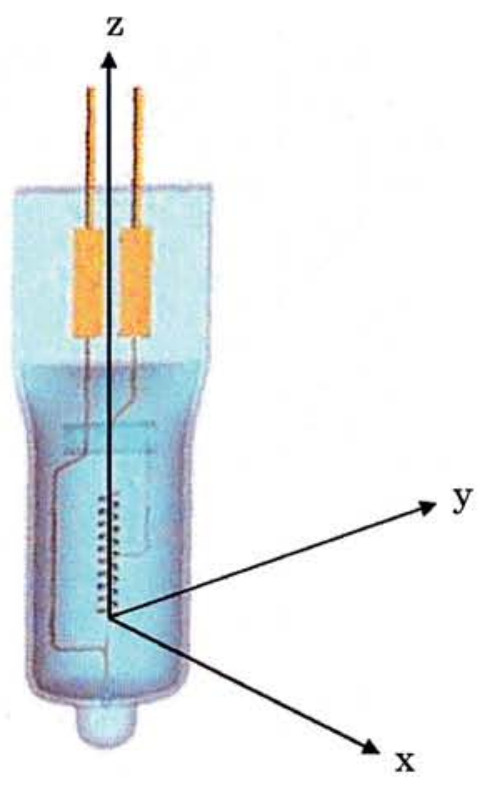

Figure 2 Definition of coordinate axes
Moreover, a comparison to the case in which a coil lead is attached to both ends reveals a significant difference not only in the distribution, but also in the flow velocity itself. These results indicate the need to reconsider the model, including the coil leads. In particular, with a model in which a coil lead is not attached, careful attention must be paid so that flow stagnation does not occur at the coil ends. Also, in actuality, the effect of a chemical attack occurring due to contamination from $\mathrm{H}_{2} \mathrm{O}$ or other impurities is prominent in low temperature areas. For these reasons, we decided to include the coil leads in the model. In Figure 3(a) and (b), the filament of the light emitting part and the coil leads are shown in white. In Figure 3(b), arrows on the inner side of the filament coil overlap and are difficult to see, but the orientation of the blue arrows $\left(1.67 \times 10^{-2}\right.$ to $3.35 \times 10^{-2} \mathrm{~m} / \mathrm{sec}$ ) is upward. Also, the orientations of the blue-colored arrows $\left(2.45 \times 10^{-5}\right.$ to $\left.5.60 \times 10^{-3} \mathrm{~m} / \mathrm{sec}\right)$ are along the cross-section of the coil, and orientations appear to be disordered. Figure 3(c) shows an extracted view of the center part of the filament, with the density of the arrows having been changed.

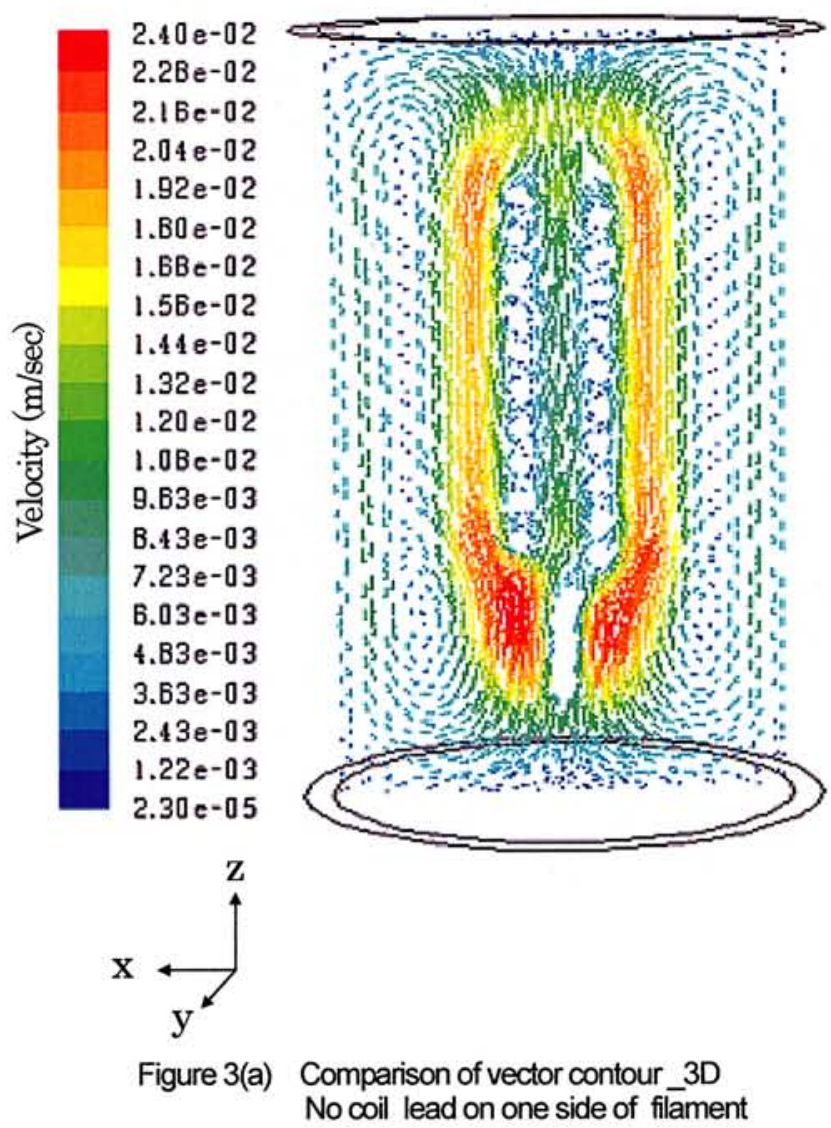




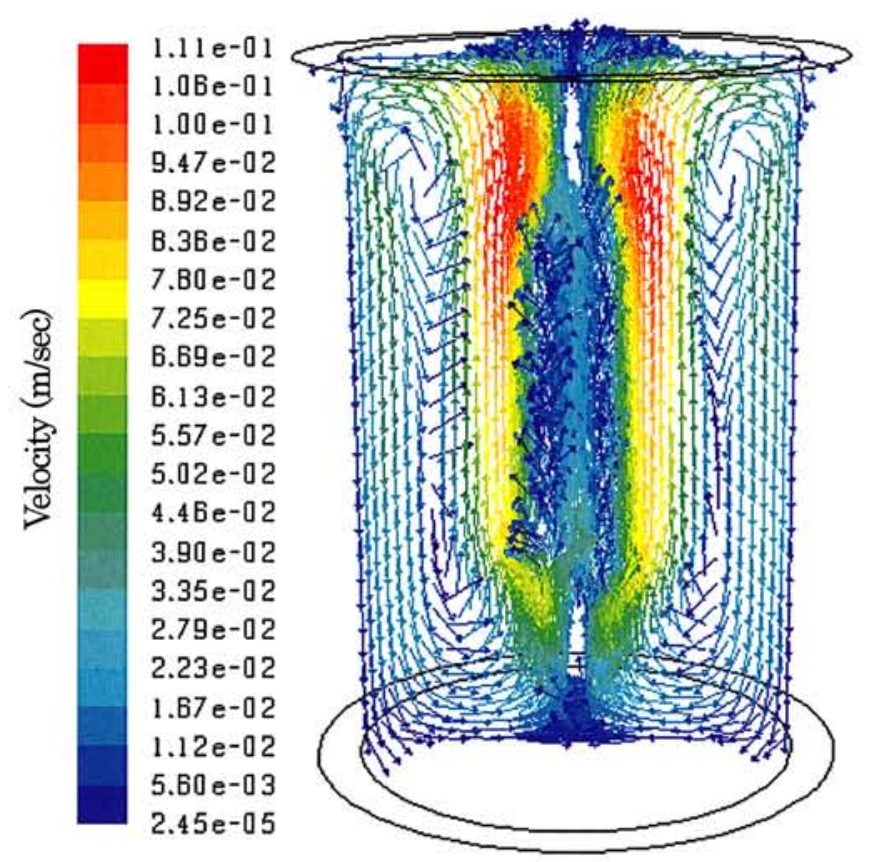

$\mathrm{x}$

Figure 3(b) Comparison of vector contour_3D Coil lead attached to both sides of filament

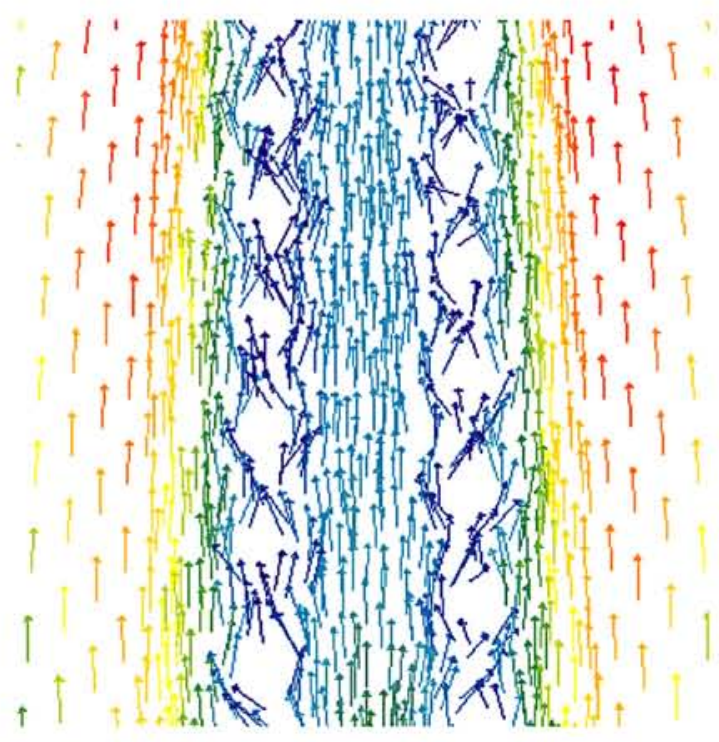

Figure 3(c) Comparison of the vector contour 3D Magnified view of œnter part of filament

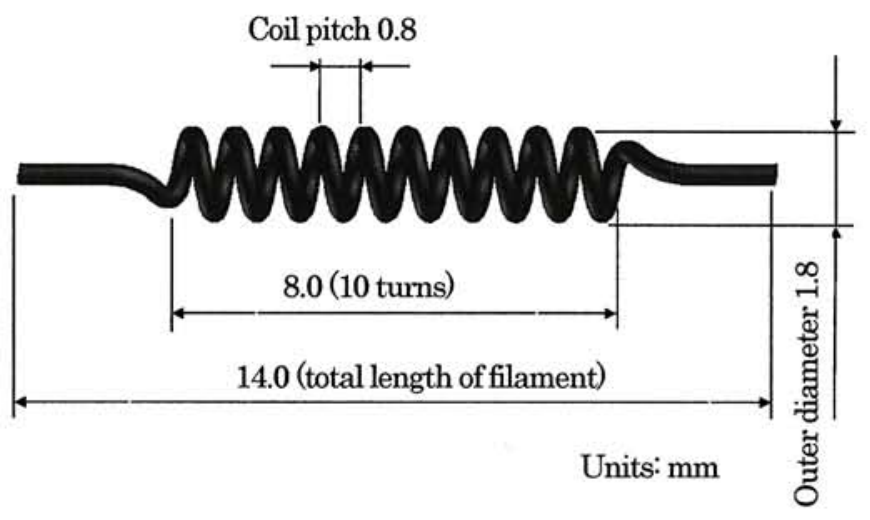

Figure 4 Filament dimensions

Figure 4 shows the shape of the coil used in the analysis. The actual filament is a double coil, but a double coil is difficult to model with $\mathrm{CAD}$, and even if a model was created, the surrounding mesh would have be made extremely small, and an extremely long time would be required for the iterative calculations of the chemical equilibrium, and therefore modeling of a double coil is impractical. Accordingly, in this analysis, the filament is considered to be a single coil in the calculations. The coil pitch is $0.8 \mathrm{~mm}$, and assuming that the single coil part of the filament has a cylindrical shape, the surface area of the coil is $6.00 \times 10^{-5} \mathrm{~m}^{2}\left(60 \mathrm{~mm}^{2}\right)$.

\subsubsection{3-D analysis mesh}

The following type of $3-\mathrm{D}$ analysis mesh was set for the tube.

- Bulb shape

A quartz casing having an inner radius of $5.00 \times 10^{-3} \mathrm{~m}(5$ $\mathrm{mm})$ and an outer radius of $6.00 \times 10^{-3} \mathrm{~m}(6 \mathrm{~mm})$ was set as the bulb area. Also, the bulb shape was approximated with a cylinder of $1.60 \times 10^{-3} \mathrm{~m}(16 \mathrm{~mm})$.

- Analysis mesh

Tetrahedral and hexahedral primary elements of 168,145 nodes and 283,140 elements were set in the interior of the bulb. To increase the calculation accuracy, hexahedral elements are preferred, but because the filament shape is thin and complex, only the periphery of the filament was subdivided into tetrahedral elements.

- Physical properties

The physical attributes of a $\mathrm{Kr} / \mathrm{N}_{2}$ mixed gas were set for the interior of the casing and the physical attributes of quartz were set for the casing. 


\subsubsection{Thermo fluid boundary condition settings}

The boundary conditions were set as follows for the calculations.

- Heat source

The heat source is the thermal radiation from the surface of the filament, and the amount of heat generated is $85 \mathrm{~W}$. If the efficiency of conversion to visible light is $10 \%$, this amount of heat generation is equivalent to a $100 \mathrm{~W}$ class halogen lamp. This amount of heat generation, when converted into the heat generation per unit area, i.e., the thermal flow rate, is $1.42 \times 10^{6} \mathrm{~W} / \mathrm{m}^{2}$.

- Features of the inner and outer surfaces of the casing (quartz bulb)

Setting the thermal transmittance and reflective boundary conditions for the inner casing surface enables both the transmittance and reflectivity of thermal radiation at the boundary surface to be considered. Also, the scattering coefficient is set to 0.05 so that the effects of roughness, dirt and the like on the inner surface of the glass casing are included in the analysis.

- Thermal conductivity of quartz glass

The thermal conductivity of the quartz glass was set to $1.6 \mathrm{~W} / \mathrm{m} \cdot \mathrm{K}$.

- Average molecular weight for thermo fluid field analysis In the calculation of the convective velocity field, a gas mixture of $\mathrm{Kr}: 90 \% \mathrm{~N}_{2}: 10 \%$ was assumed and the average molecular weight of $78.2 \mathrm{~g} / \mathrm{mol}$ was used as the molecular weight of the gas.

- Heat transfer coefficient of quartz glass

In this analysis, in order to lessen the computational load, instead of setting a calculation area for the outside of the lamp, the heat transfer coefficient of the glass surface was adjusted to find conditions whereby the outer surface temperature of the bulb of the halogen lamp approaches the actual temperature. The purpose of the analysis is to examine phenomena inside the tube, and as long as the temperatures of the filament and bulb areas are reproduced accurately, they can be used as the boundary conditions without any special restrictions.

- Boundary conditions for outer surface of bulb Recently, the outer surface of the glass is often coated with an infrared reflective film. In the case where the glass is coated with an infrared reflective film and the physical properties of the quartz glass are used as is, the surface temperature may not be calculated accurately as actual surface temperature readings. Thus, in this analysis, to ensure the versatility of the model, the heat transfer coefficient of the outer surface of the bulb is adjusted, reproducing the natural thermal transmittance and radiation generated from the lamp, and conditions capable of successfully reproducing the temperature distribution and the like of the outer surface of the bulb were employed. By setting the heat transfer coefficient to $200 \mathrm{~W} / \mathrm{m}^{2} \mathrm{~K}$ with the thermal transmittance and reflective boundary conditions, and setting the scattering coefficient to 0.05 which is the same value as for the inner surface, as shown in Figure 5, the reproduction of the distribution of surface temperature of an actual bulb was verified to be good. Although the heat transfer coefficient and scattering coefficient values set here are assumed values, if, as the result of the thermo fluid calculations, the surface temperature of the bulb can reproduce the actual temperature distribution, the boundary condition settings are deemed to be correct.

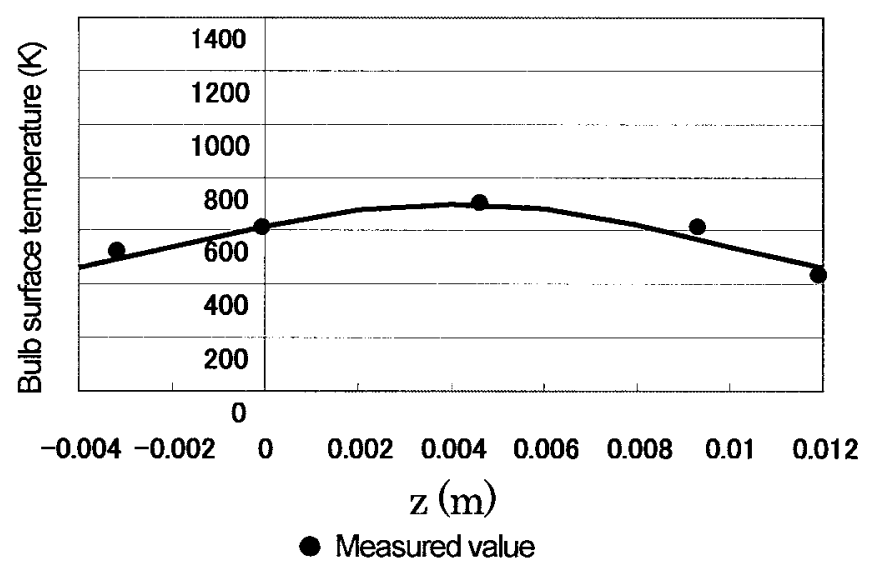

Figure 5 Temperature distribution of the bulb surface

The temperature distribution at the $y=0$ plane is shown in Figure 6(a). As in the velocity distribution shown in Figure 3, this temperature distribution is nearly axissymmetric. A two-dimensional model was used when analyzing the chemical equilibrium and performing complex calculations.

The temperature distribution of an actual filament is shown in Figure 6(b) and is in good agreement with the simulated results. The end tips of the coil leads in Figure 6(a) correspond to the end tips of the coil leads of the actual halogen lamp shown in Figure 6(b). In an actual halogen lamp, since the coil leads are not coils but instead are element wires, the thermal conductivity is poor, and as a result, the temperature gradient becomes large and the temperature drops to 1250 to $1260 \mathrm{~K}$ in the vicinity of the support connections. For the simulation, the coil of the halogen lamp shown in Figure 6(b) was modeled from the upper coil lead to the lower coil lead.

\subsection{Condition settings for the thermo fluid-chemical equilibrium hybrid model}

3.3.1 Two-dimensional velocity field and temperature field

The analysis mesh used for the calculations is shown in Figure 7(a). Although this mesh is three-dimensional, the thickness is $1 \mathrm{~mm}$ and since there is only one element in 

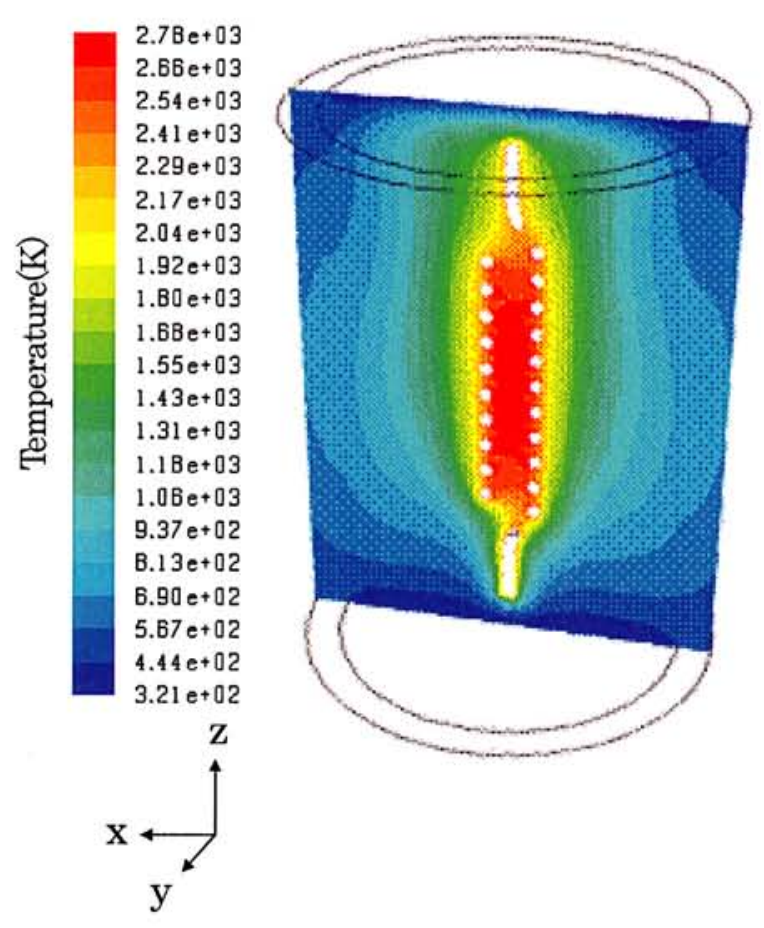

Figure 6(a) Temperature distribution 3-D simulation of temperature distribution

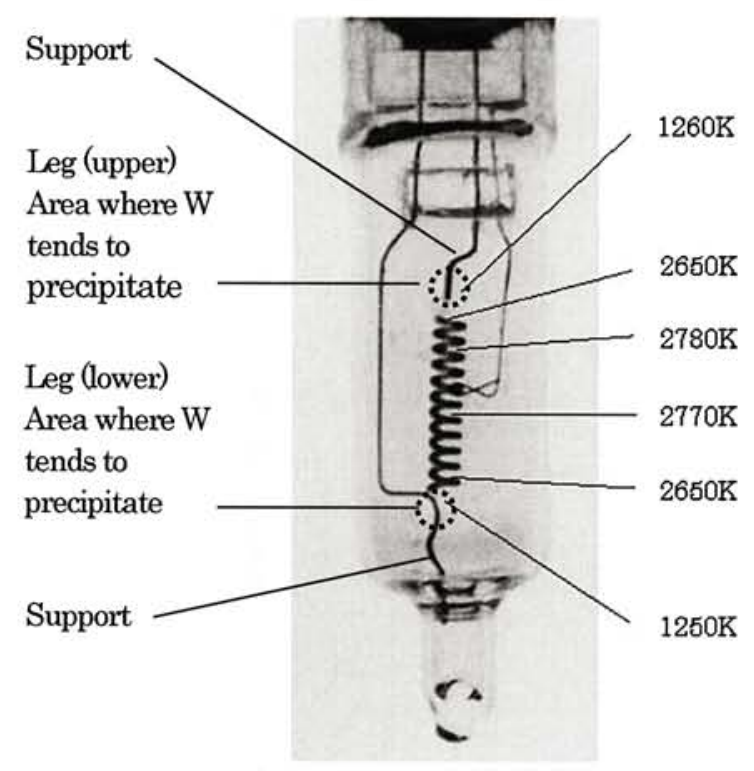

Figure 6(b) Temperature distribution Measured values

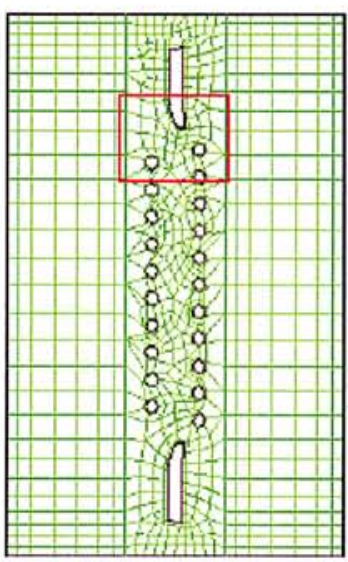

(a) Analysis mesh

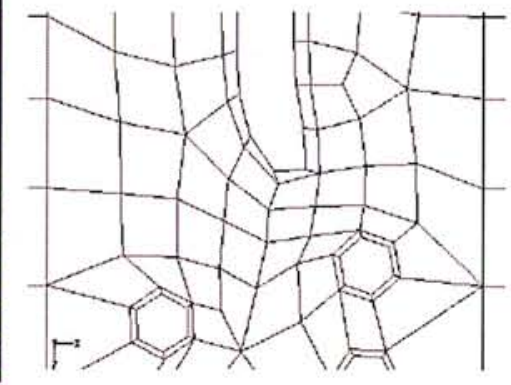

(b) Enlarged view of main section
Figure 7 Mesh of the model

the thickness direction, the model is essentially twodimensional. An enlarged view of the area inside the red frame in the figure is shown in Figure 7(b). The mesh is set to be particularly fine for cells that border the crosssection of the filament.

If the velocity field of the $y=0$ cross-section obtained by three-dimensional model analysis is simply applied to a two-dimensional model, the flow will no longer satisfy the continuity equation. Therefore, the flow field was recalculated so that the flow field would satisfy the continuity equation and that the temperature distribution would be within \pm 10 degrees of the actual measured values. The usual fluid calculations are performed, but an external force known as a penalty term $f_{p}$ is applied so that the flow field approaches the target value (calculated value obtained with a three-dimensional model). The penalty term $f_{p}$ is a body force that makes an unsteady calculated flow field approach its target value, and is given by the following equation:

$$
f_{p}=\alpha\left(v_{c}-v_{o}\right)
$$

Here, $v_{c}$ is the value calculated with a two-dimensional model, and $v_{o}$ is the target value. $\alpha$ is chosen such that velocity field values close to those of the three-dimensional model can be obtained. There are no general guidelines for the allowable error, and visual assessments are made while comparing the velocity vectors. However, the profile of the flow velocity changes significantly according to $\alpha$, and the freedom of choice is considered to be small. Since the recalculated flow field satisfies the continuity equation, this flow field is used to carry out unsteady, twodimensional calculations of the changes in concentrations of the substances. The recalculated results are shown in Figure 8 . The maximum flow rate is $1.10 \times 10^{-1} \mathrm{~m} / \mathrm{sec}$ and the minimum flow rate is $4.65 \times 10^{-5} \mathrm{~m} / \mathrm{sec}$. With the original three-dimensional model, the maximum flow rate 
is $1.29 \times 10^{-1} \mathrm{~m} / \mathrm{sec}$ and the minimum flow rate is $1.77 \times 10^{-4}$ $\mathrm{m} / \mathrm{sec}$, and values very close to these have been reproduced. The velocity field is slightly disturbed at areas that collide with the filament, but this is because the flow is limited to two dimensions and there is no escape for the flow. However, except for the flow field extremely close to the filament, nearly the same profile as the threedimensional analysis results can be obtained. Similar results can be obtained for the temperature field.

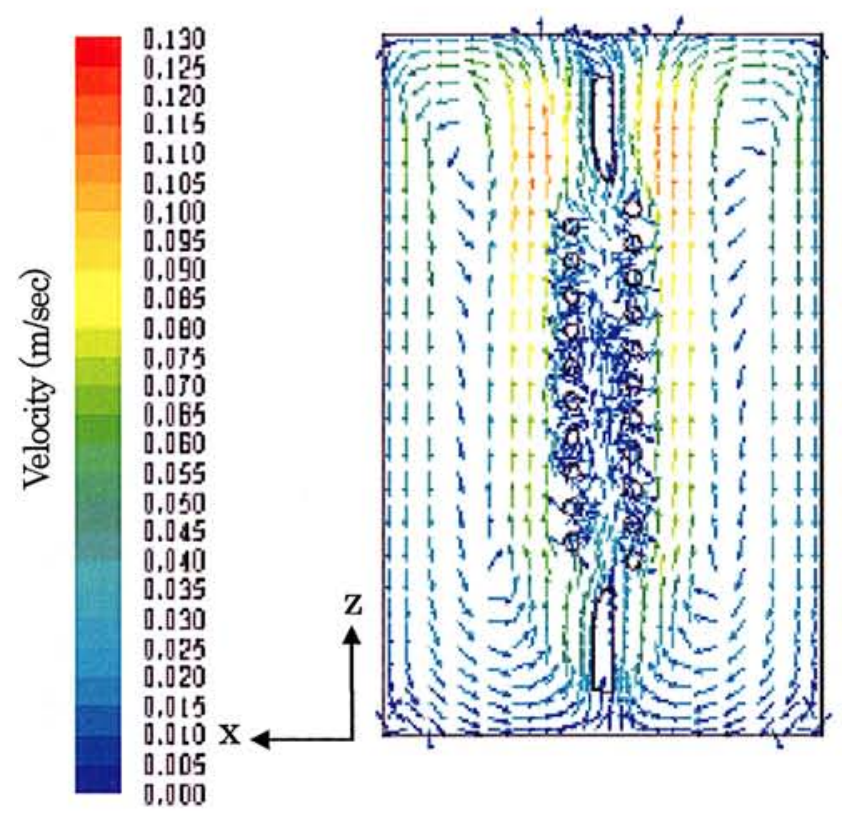

Figure 8 Vector contour in 2-D model

\subsubsection{Chemical equilibrium calculations}

The chemical equilibrium calculations were carried out using FactSage ${ }^{\mathrm{TM}}$ and the ChemApp equilibrium calculation library and by linking to Fluent ${ }^{\circledR}$. The SGTE thermodynamic database was used for the equilibrium calculations. The input values required for the equilibrium calculations are the chemical composition, temperature and pressure. Chemical equilibrium calculations were carried out for all cells in $0.5 \mathrm{sec}$ time steps. Next, the reacting quantity (reacting weight) $R_{i}$ of the chemical species $i$ that actually reacts in each cell is calculated. The reacting weight $\boldsymbol{R}_{i}$ is defined using the reaction ratio $\boldsymbol{r}_{\boldsymbol{r}}$, and the amount of the chemical species that reacts was controlled. The reacting quantity is specified in $\mathrm{kg}$ units and is given by the following equation as a function of temperature and the mass of chemical species in the cell:

$$
R_{i}=r_{r} \times m_{i}(x)
$$

where, $m_{i}$ is the mass $(\mathrm{kg})$ of chemical species $i$ in the cell, $A_{R}$ is a coefficient to control reaction rate, and the units are in the Kelvin temperature scale.

Also, the reaction ratio $r_{r}$ is given by the following equation.

$$
r_{r}=\exp \left(-\frac{A_{\mathrm{R}}}{T}\right)
$$

The concept of the reaction rate is not included in equilibrium theory, and therefore the reacting weight is defined as above, based on the Arrhenius equation.

Also, relevant literature about the reaction rate control method could not be found, and therefore the constant $A_{R}$ was selected such that the reaction ratio for hightemperature parts would be approximately $30 \%$. The $30 \%$ reaction ratio is an assumption, but as will be described later, because the time until stabilization of the partial pressure distribution is sufficiently short compared to the service life of the halogen lamp, this value of the reaction ratio is not expected to affect the stabilization time of the partial pressure distribution. Figure 9 shows the calculated results of the reaction ratio for $A_{R}=3000 \mathrm{~K}$, and the reaction ratio at high-temperature parts is $33.5 \%$. However, $r_{r}=1$ only when calculating the initial state. The condition of $r_{r}=1$ implies equilibrium, and only in the initial state $(t=1 \mathrm{sec})$ is there assumed to be a chemical equilibrium in each cell.

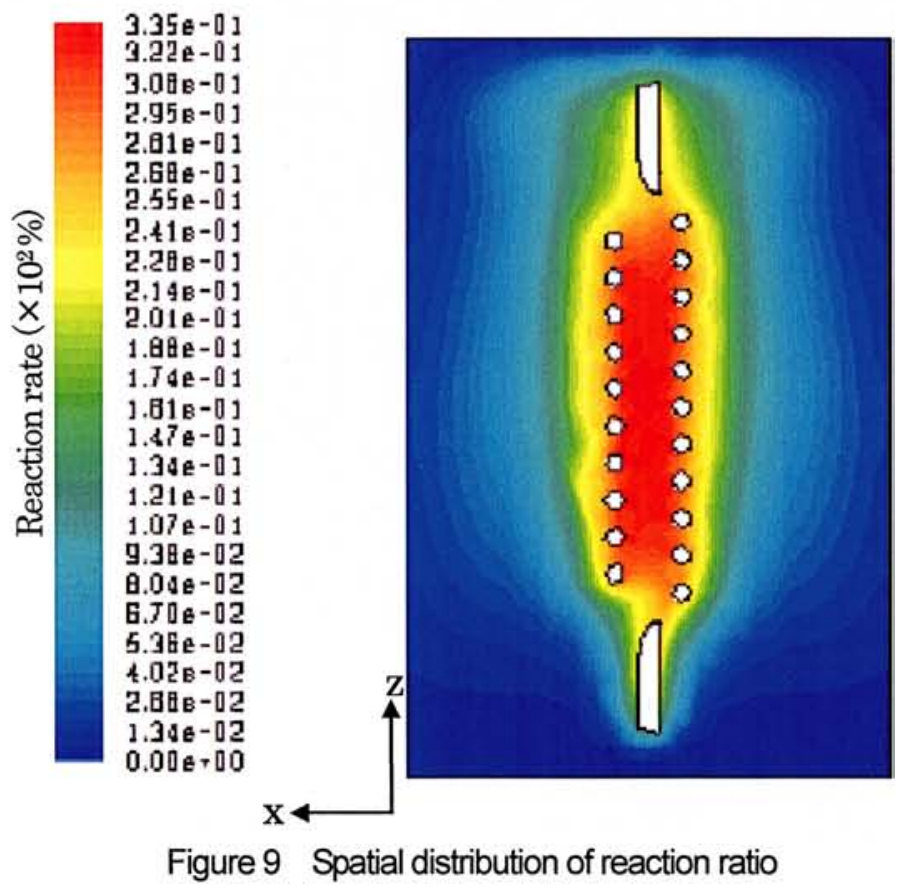


Table 1 lists substances for which the equilibrium calculations and time change of concentration are considered, and Table 2 lists the initial filling composition. In the initial state, $\mathrm{W}$ is present only in cells adjacent to the filament, and is treated as always being present in the same amount. Because Fluent ${ }^{1}$ is limited to handling up to 50 species, the equilibrium calculations were carried out assuming a total of 45 species, which consisted of the 44 species expected to be produced in the largest amounts plus the initial filling substance of $\mathrm{CH}_{2} \mathrm{Br}_{2}$. In the initial state, the system included small amounts of $\mathrm{O}_{2}$ and $\mathrm{H}_{2} \mathrm{O}$, but these amounts are residue levels from the actual lamp manufacturing process, and are not at levels that would cause trouble such as a shorter service life or blackening. Moreover, in tests where a lamp has deliberately been filled with a halogen gas mixture containing these amounts of $\mathrm{O}_{2}$ and $\mathrm{H}_{2} \mathrm{O}$, the lamp has been confirmed to have a normal service life.

Various chemical species are produced inside the bulb, but no universal relation exists for determining the value of the coefficient of molecular diffusion $D_{i m}$ for the mixture $\boldsymbol{m}$ of the chemical species $\boldsymbol{i}$ contained in Equation (4).

Therefore, the following general estimation equation from reference document (25) was used in this calculation.

$$
D_{i m}=\frac{\exp (\ln A+s \ln T)}{p}
$$

Here, $p$ is pressure, and $A$ and $s$ are constants that were set as $A=0.653 \times 10^{-5}$ and $s=1.766$. Because data pertaining to $A$ and $s$ in a halogen cycle could not be found, the numeric values given in reference literature (25) were also used here. $T$ is the temperature of each cell. $D_{\text {im }}$ does not depend on the chemical species $i$, and was assumed to take the same value for all chemical species.

\section{Results}

The following are examples of partial pressure distributions calculated based on the results of the aforementioned considerations. From among the compounds that may affect the mechanism of tungsten deposition, $\mathrm{Br}_{2}, \mathrm{~W}$ and SPW were selected, and the partial pressure distributions were calculated for the times of $t=$ $1 \mathrm{sec}$ (immediately after lighting) and $t=300 \mathrm{sec}$ (after stabilization). Here, SPW is a number that expresses the total amount of tungsten content per unit volume in terms of the partial pressure, and is an abbreviation of "Summed pressure of W compounds." For all compounds, at time $t=$ $300 \mathrm{sec}$ after lighting, the change in partial pressure is within the threshold value $\left(10^{-3} \mathrm{~Pa}\right)$, confirming that a near steady-state is reached.
Table 1 List of species

\begin{tabular}{|c|c|c|c|}
\hline Gas phase & Molecular weight & Solid phase & Molecular weight \\
\hline $\mathrm{Kr}$ & 83.8 & W & 183.85 \\
\hline W & 183.85 & $\mathrm{WO}_{2} \mathrm{Br}_{2}$ & 375.6568 \\
\hline WO & 199.8494 & $\mathrm{C}$ & 12.011 \\
\hline $\mathrm{WO}_{2}$ & 215.8488 & $\mathrm{CW}$ & 195.861 \\
\hline $\mathrm{WO}_{3}$ & $\begin{array}{|ll|}23.2 & 8224 \\
\end{array}$ & $\mathrm{CW}_{2}$ & 379.711 \\
\hline $\mathrm{W}_{2} \mathrm{O}_{6}$ & 463.6934 & $\mathrm{WO}_{2}$ & 215.8488 \\
\hline $\mathrm{W}_{3} \mathrm{O}_{8}$ & 679.5452 & $\mathrm{~W}_{7} \mathrm{O}_{19}$ & 159.578576 \\
\hline $\mathrm{W}_{3} \mathrm{O}_{2}$ & 695.5446 & $\mathrm{BrH}_{4} \mathrm{~N}$ & 97.9425 \\
\hline $\mathrm{W}_{4} \mathrm{O}_{12}$ & 927.3928 & $\mathrm{WBr}_{4}$ & 503.466 \\
\hline $\mathrm{WBr}$ & 236.754 & $\mathrm{WBr}_{5}$ & 583.37 \\
\hline $\mathrm{WBr}_{2}$ & 343.658 & $\mathrm{WBr}_{6}$ & 663.275 \\
\hline $\mathrm{WBr}_{3}$ & 423.562 & $\mathrm{H}_{2} \mathrm{WO}_{4}$ & 249.86348 \\
\hline $\mathrm{WBr}_{4}$ & 503.466 & & \\
\hline $\mathrm{WBr}_{5}$ & 583.37 & & \\
\hline $\mathrm{WO}_{2} \mathrm{Br}_{2}$ & 35.6568 & & \\
\hline $\mathrm{H}_{2} \mathrm{WO}_{4}$ & 249.86348 & & \\
\hline $\mathrm{HBr}$ & 80.91194 & & \\
\hline $\mathrm{H}_{2} \mathrm{O}$ & 18.01528 & & \\
\hline $\mathrm{HO}$ & 17.00734 & & \\
\hline $\bar{C}$ & 12.011 & & \\
\hline $\mathrm{CO}$ & 28.0104 & & \\
\hline $\mathrm{CO}_{2}$ & 44.0098 & & \\
\hline $\mathrm{Br}$ & 79.904 & & \\
\hline $\mathrm{Br}_{2}$ & 159.808 & & \\
\hline $\mathrm{BrN}$ & 93.91074 & & \\
\hline $\mathrm{N}_{2}$ & 28.01348 & & \\
\hline $\mathrm{NO}$ & 30.00614 & & \\
\hline $\mathrm{NO}_{2}$ & 46.00554 & & \\
\hline $\mathrm{O}$ & 15.9994 & & \\
\hline $\mathrm{O}_{2}$ & 31.9988 & & \\
\hline $\mathrm{H}$ & 1.0794 & & \\
\hline $\mathrm{H}_{2}$ & 2.01588 & & \\
\hline
\end{tabular}

Table 2 Filling amount of the initial state

\begin{tabular}{|c|c|}
\hline Substance & Fraction \\
\hline $\mathrm{Kr}$ & $9.00 \times 10^{-1}\left(9.00 \times 10^{5} \mathrm{ppm}\right)$ \\
\hline $\mathrm{N}_{2}$ & $9.93 \times 10^{-2}\left(9.93 \times 10^{4} \mathrm{ppm}\right)$ \\
\hline $\mathrm{CH}_{2} \mathrm{Br}_{2}$ & $6.00 \times 10^{4}\left(0.60 \times 10^{3} \mathrm{ppm}\right)$ \\
\hline $\mathrm{O}_{2}$ & $5.00 \times 10^{-5}\left(0.50 \times 10^{2} \mathrm{ppm}\right)$ \\
\hline $\mathrm{H}_{2} \mathrm{O}$ & $2.00 \times 10^{-5}\left(0.20 \times 10^{2} \mathrm{ppm}\right)$ \\
\hline
\end{tabular}




\subsection{Transition of $\mathrm{Br}_{2}$ partial pressure distribution}

$\mathrm{Br}_{2}$ is produced by the decomposition of $\mathrm{CH}_{2} \mathrm{Br}$, which is the initial filling substance. Figure 10 shows the partial pressure distribution of $\mathrm{Br}_{2}$ immediately after lighting and after the partial pressure of each chemical species has stabilized. Immediately after lighting at $t=1 \mathrm{sec}$, the partial pressure distribution still remains in the state prior to the effects of convection and diffusion.

According to previous reports, at temperatures of about $1600 \mathrm{~K}$ or higher, $\mathrm{W}$ and $\mathrm{Br}_{2}$ do not produce much compounds and instead coexist in mutually separate forms. This interpretation describes $\mathrm{W}$ as returning to high-temperature parts of the filament ${ }^{2}$. In the results of this simulation shown in Figure 10, at $t=1 \mathrm{sec}$, the partial pressure of $\mathrm{Br}_{2}$ is highest, on the order of $10^{-1} \mathrm{~Pa}$, at approximately $1600 \mathrm{~K}$, while in the vicinity of even higher temperature parts of the filament, the partial pressure is on the order of $10^{2} \mathrm{~Pa}$, which is a rather lower level.

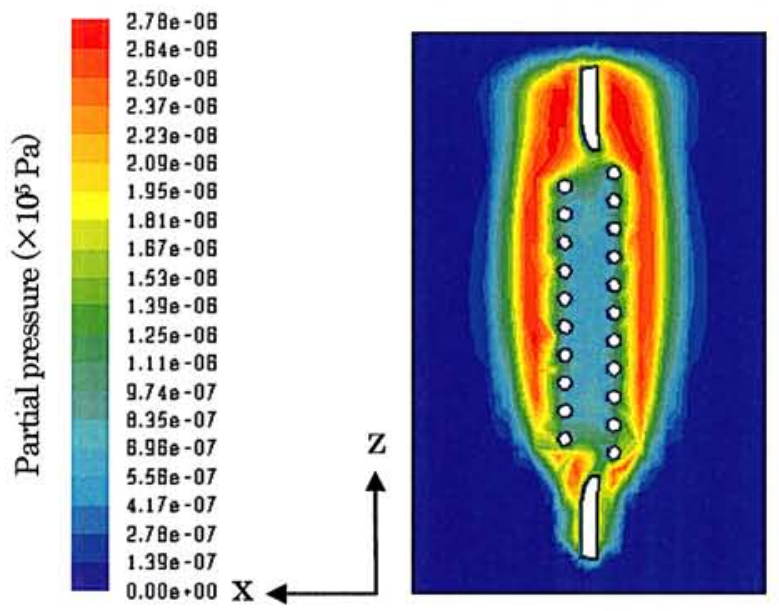

(a) Partial pressure distribution at $t=1 \mathrm{sec}$

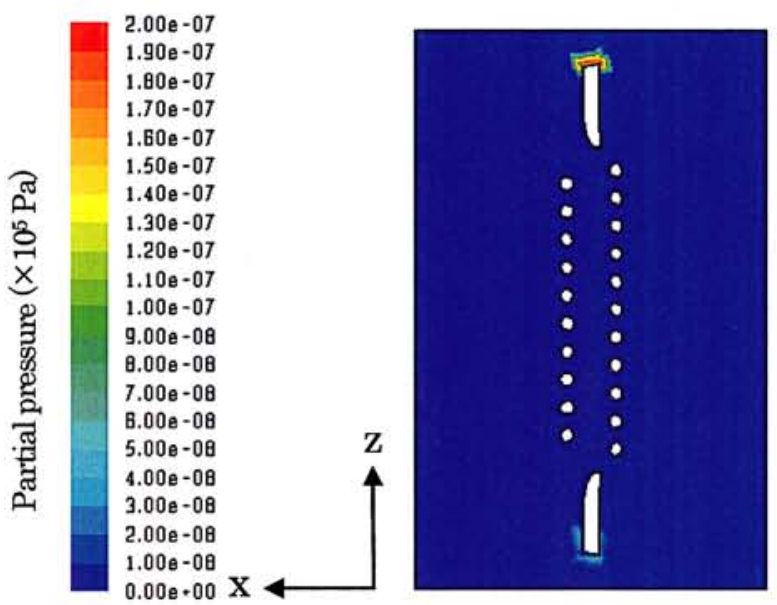

(b) Partial pressure distribution at $t=300 \mathrm{sec}$

Figure 10 Transition of $\mathrm{Br}_{2}$ partial pressure
However, after the partial pressure has stabilized at $t=$ $300 \mathrm{sec}$, the upper leg is the site of the highest $\mathrm{Br}_{2}$ partial pressure which is on the order of $10^{-2} \mathrm{~Pa}$. The distribution of partial pressure may also be affected by convection and thermal diffusion and is not unequivocally determined by temperature, and the partial pressure may differ even at locations of the same temperature. The partial pressure of $\mathrm{Br}_{2}$ in the vicinity of the filament is lower by at least two orders of magnitude, and the $\mathrm{Br}_{2}$ that functions substantially in the halogen cycle is concentrated at locations in the periphery of the coil leads.

\subsection{Transition of $\mathrm{W}$ partial pressure distribution}

$\mathrm{W}$ is vaporized from the filament and released in a gaseous phase. Fig 11 shows the partial pressure distribution of $\mathrm{W}$ immediately after lighting and after the partial pressure of each chemical species has stabilized. Immediately after lighting at $t=1 \mathrm{sec}$, the vapor pressure of $\mathrm{W}$ in the vicinity of the filament is high because most of the vaporized $\mathrm{W}$ remains and has not yet been transported by convection or diffusion.

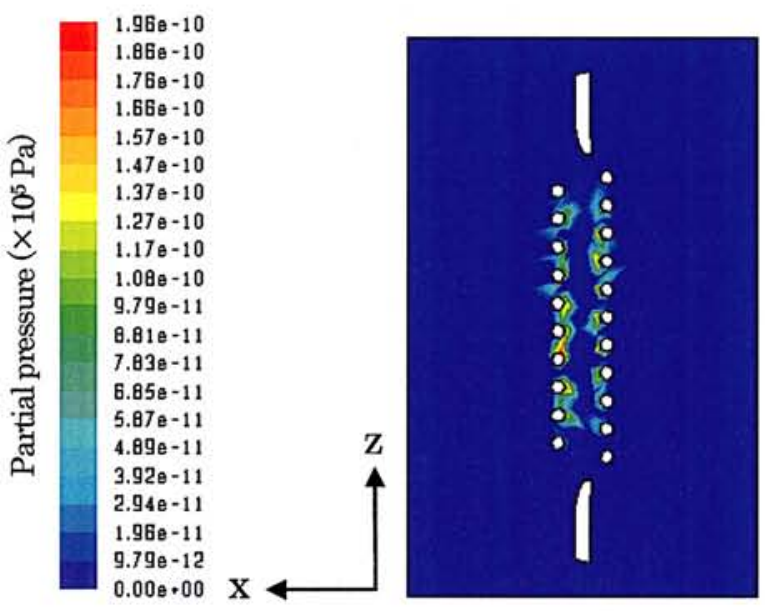

(a) Partial pressure distribution at $t=1 \mathrm{sec}$

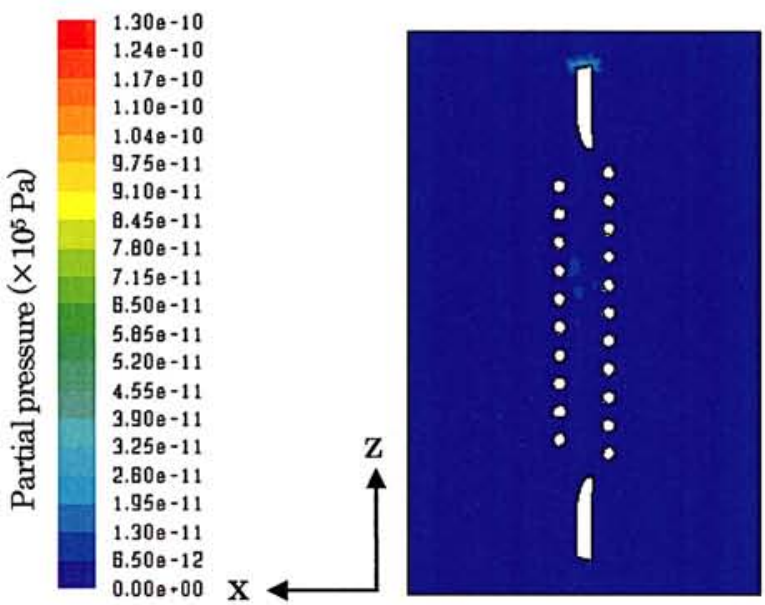

(b) Partial pressure distribution at $t=300 \mathrm{sec}$

Figure 11 Transition of W partial pressure 
However, with the passing of time, $\mathrm{W}$ gathers in the periphery of the upper coil lead, and when the partial pressure distribution stabilizes, the $\mathrm{W}$ partial pressure is highest in this area. Also at the center part of the filament, areas remain with partial pressures that are similar to those in the periphery of the upper coil lead, but compared to the conditions at $t=1 \mathrm{sec}$, these partial pressures are an order of magnitude lower. The change in the $\mathrm{W}$ partial pressure over time is caused by the migration of the vaporized $\mathrm{W}$ by convection and diffusion. The periphery of the coil leads is at a low temperature of approximately $1200 \mathrm{~K}$, but with a chemical equilibrium model that does not account for convection, this type of migration phenomenon cannot be reproduced and therefore the same W partial pressure as at $t=1 \mathrm{sec}$ is obtained. With a hybrid model, however, the $\mathrm{W}$ partial pressure in the vicinity of the filament is low, and producing almost no $\mathrm{Br}$, $\mathrm{O}$ or other compounds, the vaporized $\mathrm{W}$ is expected to be transported to the periphery of the coil leads.

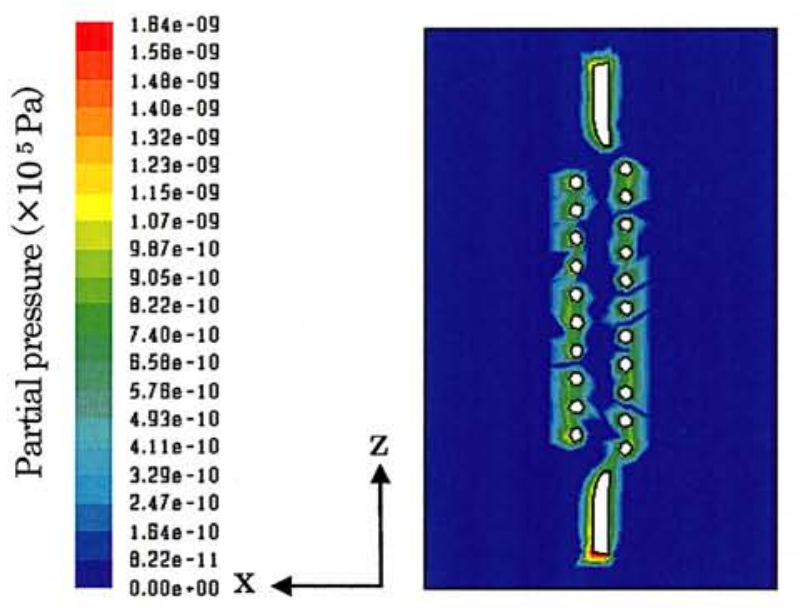

(a) Partial pressure distribution at $t=1 \mathrm{sec}$

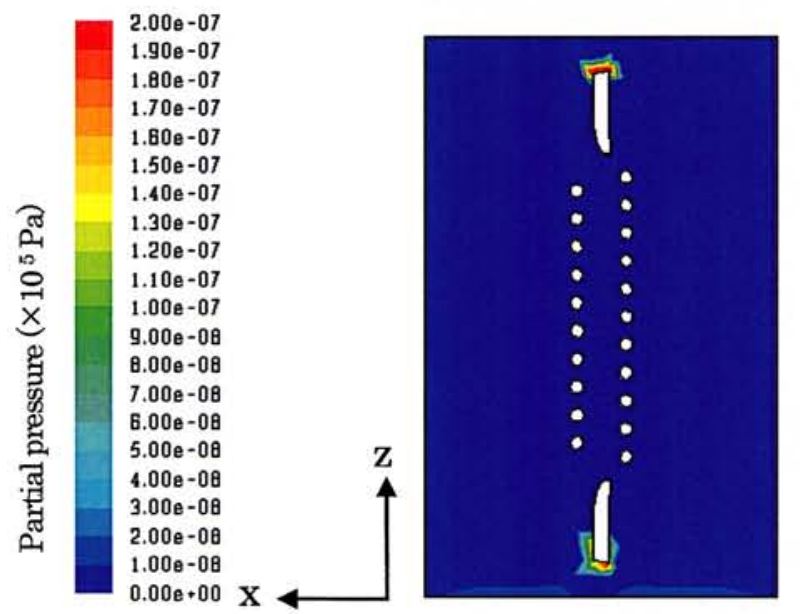

(b) Partial pressure distribution at $t=300 \mathrm{sec}$

\subsection{Transition of SPW partial pressure distribution}

The SPW is a number that expresses the total amount of tungsten content per unit volume in terms of the partial pressure, and accordingly, locations having a high SPW partial pressure also have a high density of W. Figure 12 shows the SPW partial pressure distribution immediately after lighting and after the partial pressure of each chemical species has stabilized. While assuming the form of various compounds, $\mathrm{W}$ moves around the interior of the bulb, and tungsten was found to be present in large quantities at the coil leads on both ends of the filament and at low temperature areas on the inner wall of the bulb. The SPW value is higher at the inner wall of the bulb due to the production of $\mathrm{WO}_{2}(\mathrm{~s})$.

In previous studies based on chemical equilibrium theory, the SPW value decreased at intermediate areas between the tungsten incandescent region and low temperature areas such as the inner wall of the bulb, and $\mathrm{W}$ precipitated in such areas. However, with the hybrid model of this study, the SPW value is low at nearly all regions in the bulb interior but is high at the ends of both coil leads, and a situation arises which is different from that of the conventional model. Moreover, at the bulb inner wall and other locations where the SPW value is low, blackening and tungsten precipitation were not observed.

\section{Discussion}

\subsection{Conventional model}

Based on chemical equilibrium calculations, Dr. Neumann summarized the halogen cycle process as the diagrammatic representation shown in Figure $13^{8}$.

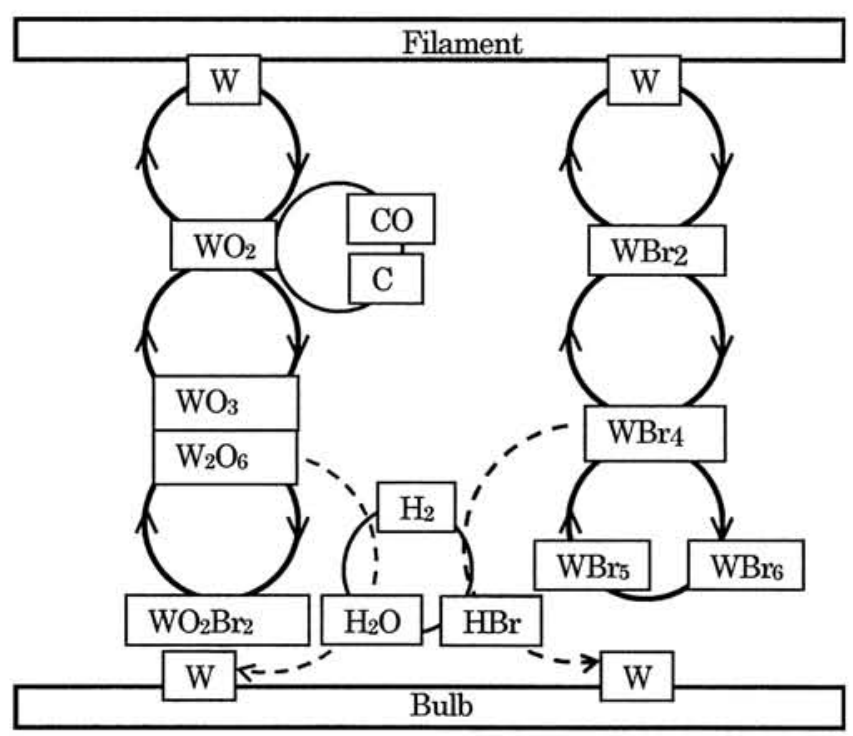

Figure 13 Tungsten-halogen cycle in the equilibrium model 
The halogen cycle reaction consists of two serial reactions, $\mathrm{W}-\mathrm{Br}$ and $\mathrm{W}-\mathrm{O}-\mathrm{Br}$. The $\mathrm{W}-\mathrm{Br}$ and $\mathrm{W}-\mathrm{O}-\mathrm{Br}$ compounds formed by these reactions cause the "dissolution" of $\mathrm{W}$ and act as a mechanism that prevents blackening. However, if a $\mathrm{C}$ compound is present, particularly in a high temperature region, $\mathrm{O}$ is taken from the $\mathrm{W}-\mathrm{O}-\mathrm{Br}$ series reaction to form $\mathrm{CO}$, diminishing the function for the "dissolution" of $\mathrm{W}$ into a gaseous phase, and decreasing the amount transported. On the other hand, in low temperature regions, $\mathrm{CO}$ acts as an $\mathrm{O}$-donor and assists in the formation of $\mathrm{W}-\mathrm{O}-\mathrm{Br}$ compound to maintain the cycle reaction. Thus, in low temperature regions, $\mathrm{C}$ functions to prevent blackening.

Meanwhile, as a result of the production of $\mathrm{H}_{2} \mathrm{O}$ and $\mathrm{Br}^{-}$ $\mathrm{H}$ compounds, $\mathrm{O}$ and $\mathrm{Br}$ are taken from the series reactions, diminishing the capability for the "dissolution" of $\mathrm{W}$ into a gaseous phase, and $\mathrm{W}$ is released from the gaseous phase, forming needle-shaped crystals at the filament ends which are at a low temperature. Also, if there is an abundance of $\mathrm{H}$, blackening will occur. The cross-section of the bulb is divided into 4 reaction zones according to temperature, and the main compounds produced in each zone are shown in Figure 14.

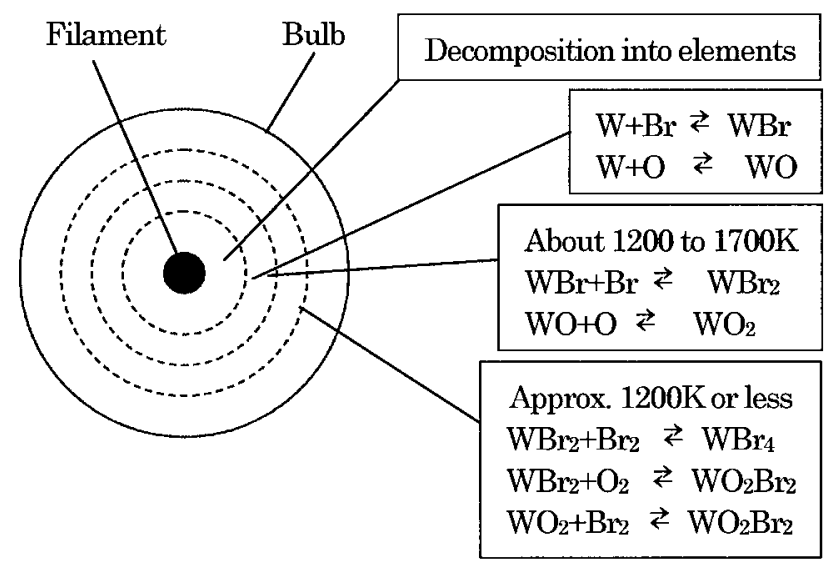

(a) Division into reaction zones

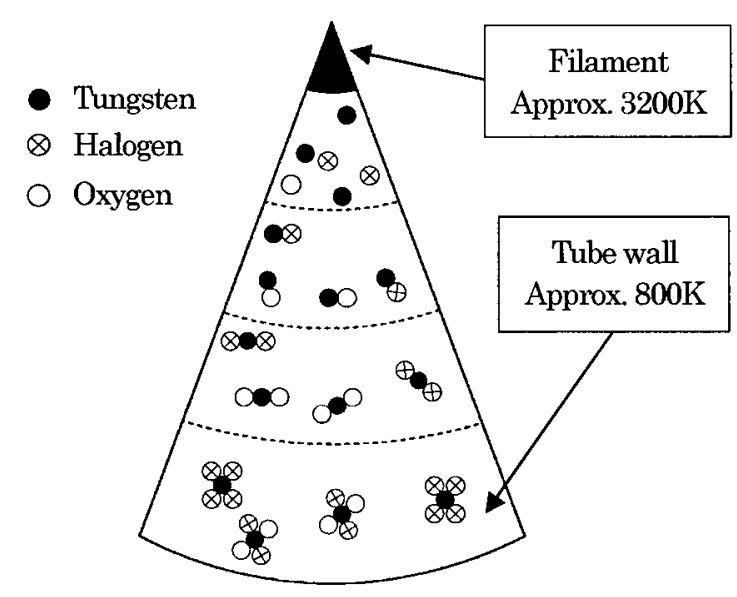

(b) Reaction zones and main compounds produced

Figure 14 Species distribution in the bulb
The temperatures for dividing the bulb interior into 4 reaction zones differ according to the design and cannot be stated concretely. What is important, however, is that the formation of aggregates in chemical equilibrium function to prevent tungsten, having vaporized from the filament, from reaching the tube wall. This "absorption" function has a larger effect than the cycle reaction that returns the vaporized tungsten back to the filament, and most of the individual reaction steps occur in the immediate vicinity of the filament.

In addition to this, many other studies of the halogen cycle have been carried out. Those studies explained that since the tungsten moves throughout the bulb interior by convection and diffusion, the tungsten will precipitate at regions of lower levels of tungsten "dissolution", i.e., in temperature zones where the SPW value is low. Also, this explanation pertains not only to halogen lamps, but is also applied to the mechanism that prevents blackening in the super high-pressure mercury lamps used in projectors ${ }^{26}$.

\subsection{Discussion of the halogen cycle mechanism}

A diagrammatic representation of the tungsten-halogen cycle inferred from the results of this study is shown in Fig. 15. There is no change from the conventional theory that the halogen cycle reaction consists of the two serial reactions of $\mathrm{W}-\mathrm{Br}$ and $\mathrm{W}-\mathrm{O}-\mathrm{Br}$. However, unlike the conventional theory in which the cycle reaction is distributed throughout the entirety of the bulb interior, this study found that the cycle reaction was concentrated in areas where convection is stagnant. Moreover, in the conventional interpretation, the "dissolution" of W throughout the entire bulb is thought to function effectively in inhibiting blackening of the halogen lamp. According to the results of this study, however, a greater effect is exhibited from the phenomenon of transport by convection from the filament periphery or bulb inner wall, and chemical species that have been transported by convection are concentrated in areas where convection is stagnant. The partial pressure distribution indicates that the tungsten-halogen cycle is actively being carried out in these stagnant regions, and that the "dissolution" of $\mathrm{W}$ occurs in these areas. Additionally, because these regions are determined by the profile of the thermo fluid, the geometrical shape of the lamp, temperature distribution, filling composition and pressure settings are important.

Together with the 2 serial reactions of $\mathrm{W}-\mathrm{Br}$ and $\mathrm{W}-\mathrm{O}-\mathrm{Br}$, $\mathrm{C}$ and $\mathrm{H}$ are also interposed in the cycle and the amounts thereof affect the partial pressure of the chemical species produced. The conventional equilibrium model does not include the concept of stagnant regions, and when the initial composition is established, the distribution of partial pressure inside the bulb is determined unequivocally. As a result, when the amount of the elements $\mathrm{H}, \mathrm{O}$ and $\mathrm{C}$ are determined, the partial pressure 


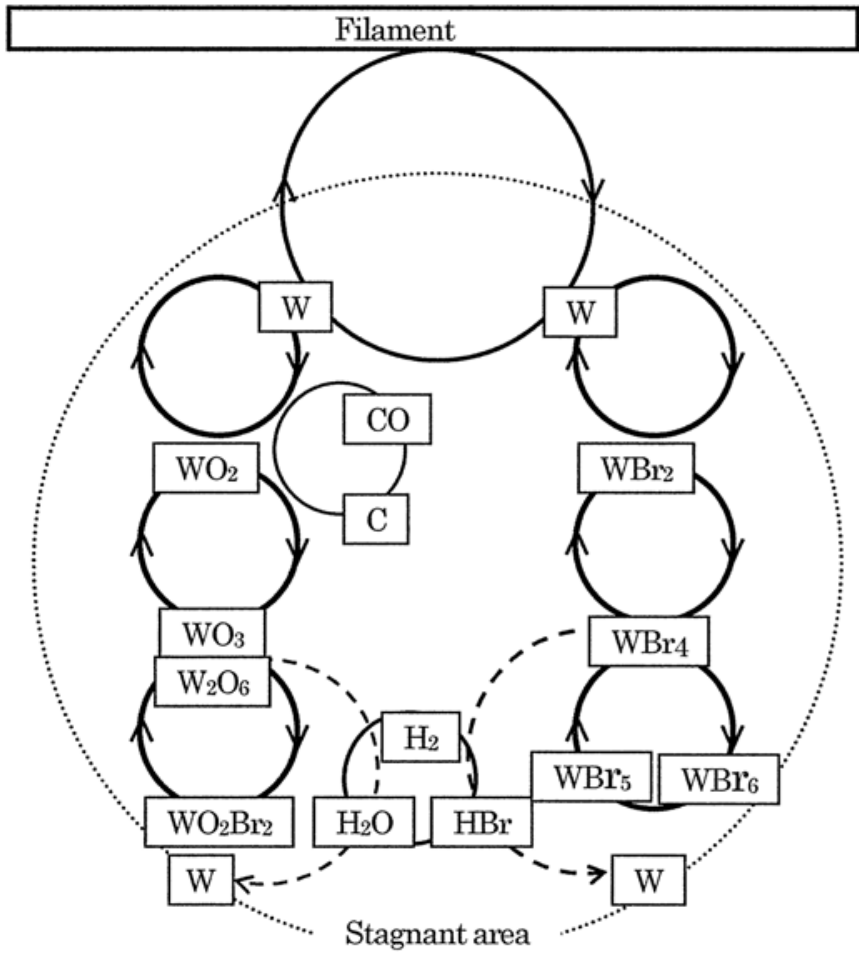

Figure 15 Tungsten-halogen cycle in the hybrid model

distribution is established, and therefore, the amounts of these elements will determine whether or not the tungsten-halogen cycle functions favorably. On the other hand, in the hybrid model of this study, stagnant regions appear, and consequently, the appropriate amount of the elements varies according to the location of the stagnant region. Thus, in the design stage, a sequence must be followed whereby, the geometric shape, the type of inert gas used as the main component of the gas, the filling pressure and the like are determined first to establish the convection state, and then the halogen and amounts of $\mathrm{H}$, $\mathrm{O}$ and $\mathrm{C}$ allowed as impurities are determined.

\subsection{Effect of impurities on the halogen cycle}

If the tungsten-halogen cycle is a mechanism such as the one proposed in Figure 15, the predicted effect of impurities remaining inside the bulb on the tungstenhalogen cycle may change. In order to predict the effect of impurities, in the lamp specifications that were calculated for this study, differences in the partial pressure distributions of the main chemical species were compared for the equilibrium theory based on Figure 13 and the hybrid calculations based on Figure 15, and the affect on the tungsten-halogen cycle was examined. Here, in contrast to the chemical equilibrium model in which the entire bulb is considered a uniform system, the hybrid model considers the effects of convection and diffusion, and consequently, the density (partial pressure) of chemical species inside the bulb is non-uniform. As a result, the partial pressure distribution differs according to the location, even when compared at the same temperatures, and even if an equal total number of moles are set for each element contained in the entire bulb, the total number of moles of chemical species contained within a fixed volume at an observed location will differ for the two models.

The temperature in the vicinity of the center of the filament is $2750 \mathrm{~K}$. The $\mathrm{W}$ vapor pressure at this point is computed to be approximately $1.2 \times 10^{2} \mathrm{~Pa}$ according to the equilibrium calculations, but is approximately $1.3 \times 10^{-6}$ $\mathrm{Pa}$ according to the hybrid calculations, and the value obtained by the hybrid calculations is much lower.

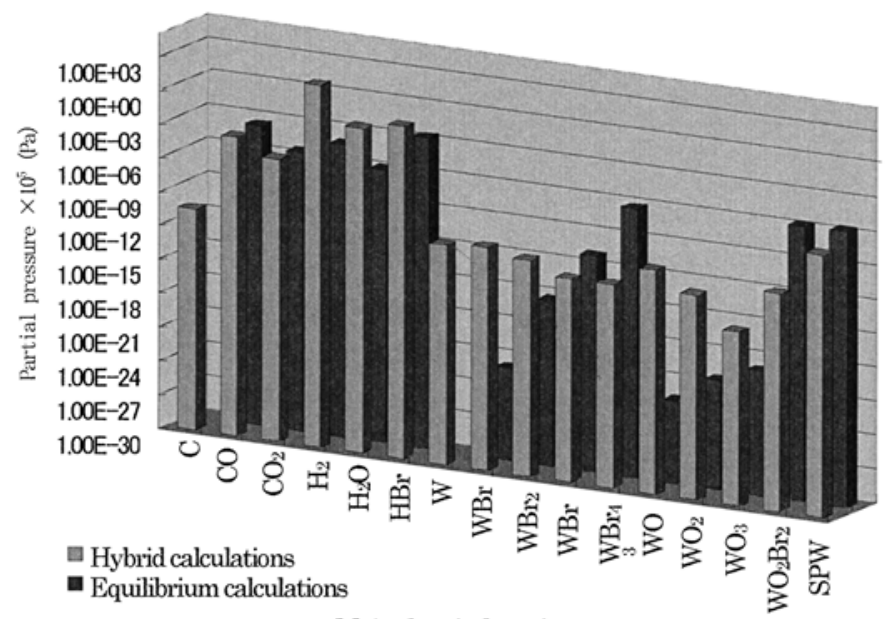

Main chemical species

Figure 16 Comparison of partial pressures

While the lamp is lighted, vaporization of $\mathrm{W}$ atoms continues for only the portion commensurate with the equilibrium vapor pressure. This amount of vaporization is determined by the filament temperature and is therefore the same for the 2 models, but the hybrid model results in a lower partial pressure because the vaporized $\mathrm{W}$ is transported by convection and diffusion.

Next, Figure 16 compares the partial pressures of the main chemical species at the end of the upper coil lead, which is at a low temperature, for results derived from the chemical equilibrium only and for results obtained from the simulation of this study. Fig. 16 shows a representative portion of the compounds produced. In contrast to the equilibrium calculations, where the entire system is considered to be a homogeneous thermodynamic system, in the calculations of this study, the assumption of thermo fluid fields resulted in the effect of the movement of chemical species, and consequently, there were 
differences in the calculated values of partial pressures.

Comparisons of the partial pressures of the chemical species constituting the $\mathrm{W}-\mathrm{O}-\mathrm{Br}$ series and $\mathrm{W}-\mathrm{Br}$ series in the vicinity of the coil leads yield the following findings.

1) Comparing the partial pressures of chemical species that constitute the $\mathrm{W}-\mathrm{O}$ series and the $\mathrm{W}$ - $\mathrm{Br}$ series, such as $\mathrm{WBr}_{2}$ and $\mathrm{WO}_{2}$ compounds, for example, in which $\mathrm{O}$ and $\mathrm{Br}$ have the same valence number with respect to $\mathrm{W}$, reveals that with the exception of WO, the partial pressure of the chemical species constituting the $\mathrm{W}-\mathrm{Br}$ series were found to be higher and the $\mathrm{W}-\mathrm{Br}$ series was dominant with the hybrid calculations.

2) For the $W$-O series, the partial pressures of chemical species such as $\mathrm{WO}$ and $\mathrm{WO}_{2}$, which have smaller $\mathrm{O}$ valence numbers, tended to be higher with the hybrid calculations than chemical species such as $\mathrm{W}_{2} \mathrm{O}_{6}$ and $\mathrm{WO}_{3}$, in which the $O$ valence number is much larger than that of $W$. These findings are contrary to those of the chemical equilibrium model.

3) For the $\mathrm{W}$-Br series as well, the partial pressures of chemical species such as $\mathrm{WBr}_{2}$ and $\mathrm{WBr}_{3}$, which have smaller $\mathrm{Br}$ valence numbers, tended to be higher with the hybrid calculations than chemical species such as WBr6, in which the $\mathrm{Br}$ valence number is much larger than that of $\mathrm{W}$. These findings are also contrary to those of the chemical equilibrium model.

The reason for these findings is thought to be due to the thermodynamic state at temperatures of 1100 to $1700 \mathrm{~K}$ and total pressures of $11 \times 10^{5}$ to $16 \times 10^{5} \mathrm{~Pa}$ at sites where the flow stagnates, and under these circumstances, the partial pressures of stable chemical species increase. From Figure 13 and Figure 15, it can be seen that for both the $\mathrm{W}-\mathrm{O}-\mathrm{Br}$ and $\mathrm{W}-\mathrm{Br}$ series, the $\mathrm{H}_{2} \mathrm{O}$ cycle acts primarily on chemical species having high valence numbers of $\mathrm{O}$ and $\mathrm{Br}$. However, the partial pressure is suppressed for chemical species having high valence numbers of $\mathrm{O}$, and therefore, even if the amount of $\mathrm{H}_{2} \mathrm{O}$ filled is increased somewhat, the overall reaction rate will become uniform for chemical species having high valence numbers of $\mathrm{O}$, and the partial pressure may not change significantly. Moreover, assuming that the $\mathrm{W}-\mathrm{Br}$ series is the main component of the halogen cycle, then even if $\mathrm{O}$ is increased, the C-O cycle will have little effect, and similar results are expected.

Next we consider findings concerning the SPW value. According to an interpretation based on the equilibrium theory, tungsten precipitates at points where the SPW value is low. Those points vary slightly according to the composition of the filling substances, but with a halogen lamp for which the proper amount of chemical species filler has been determined, SPW curve minima appear at regions of intermediate temperature between incandescent regions and low temperature areas of 1500 $K$ or less. However, with the hybrid model considered in this study, the SPW partial pressure distribution did not exhibit such minima, and on the contrary, points of higher SPW values appeared at both ends of the filament leg. The conditions for the precipitation of tungsten may be interpreted in other ways, and we wish to explore this further in subsequent reports.

\section{Conclusion}

A simulation model was created with the objective of reproducing the halogen cycle that occurs inside a lighted halogen lamp. Calculations were carried out assuming an initial filling substance comprised of $\mathrm{Kr}, \mathrm{N}_{2}$ and $\mathrm{CH}_{2} \mathrm{Br}_{2}$ and small residual amounts of $\mathrm{H}_{2} \mathrm{O}$ and $\mathrm{O}_{2}$ impurities inside the bulb, and as a result, the decomposition of $\mathrm{CH}_{2} \mathrm{Br}_{2}$ due to the lighting of the filament and production of various compounds, as well as the subsequent process in which compounds are transported by convection and diffusion inside the bulb leading to a steady state could be reproduced.

The quantity of production and decomposition, areas in which a reaction becomes active, and so on differ for each compound, and consequently, the manner of dispersion over time also differs for each product, but the partial pressure distribution of each product stabilizes at about 300 sec after lighting.

An overall characteristic is the slow convection velocity and congested flow in the vicinity of the coil leads at both ends of the filament. This is believed to be attributable to the large affect of the structure for the filling substances inside the tube, including the filament shape, and also the temperature distribution, which cause the flow to stagnate at the coil leads. This is the reason why the partial pressure of each product is high in the periphery of the coil leads, and is consistent with the areas in which tungsten precipitates at the end of the service life of the halogen lamp. In a subsequent report, while contrasting the results of real life tests and the results from the hybrid model, we plan to examine more specifically the effect of impurities on the tungsten-halogen cycle.

The model created here assumes that, at all times and all locations, equilibrium is established with the exact quantity specified by the reaction ratio. Moreover, because the boundary conditions are fixed, convection is calculated based on the assumption of a stable state. In actuality, however, approximately $300 \mathrm{sec}$ are required until the bulb reaches a stable temperature after being lighted. Simulation of the transitions during this interval would require solving unsteady equations, but this is impractical due to the burden such processing would impose on the computer processing capability. Comparison with actual phenomena would require dynamic validation to be carried out while actual measurements are taken, and we will leave this to future research. 


\section{Acknowledgements}

The authors wish to thank Osram-Melco Toshiba Lighting Ltd., Toshiba Lighting Corp., OSRAM GmbH, GTT-Technologies GmbH, and Research Center of Computational Mechanics, Inc. for their support and guidance.

\section{References}

(1) Yannopoulos, L. N. and Pebler, A.: On the Role of Oxygen, Hydrogen, and Carbon In a Tungsten Bromine Lamp, Journal of IES, pp. 21-24 (1971).

(2) Coaton, J. R.: Modern tungsten-halogen-lamp technology, IEE. Proc., 117, pp. 1953-1959 (1970).

(3) Gupta, S. K.: A Thermodynamic Investigation of the Tungsten-Oxygen-Bromine System, Journal of Physical Chemistry, 75-1, pp. 112-118 (1971).

(4) Gupta, S. K: Comments on Thermochemical Calculations of Tungsten Halogen Lamps, J. Appl. Phys., 42-13, pp. 5855-5856 (1971).

(5) Zubler, E. G. Adsorption Effects in the TungstenOxygen-Bromine Reaction, Journal of Physical Chemistry, 76-3, pp. 320-322 (1972).

(6) Dittmer, G. and Niemann, U.: HETEROGENEOUS REACTIONS AND CHEMICAL TRANSPORT OF TUNGSTEN WITH HALOGENS AND OXYGEN UNDER STEADY-STATE CONDITIONS OF INCANDESCENT LAMPS, Philips J.Res., 36, pp. 87-111 (1981).

(7) Dittmer, G. and Niemann, U.: HETEROGENEOUS REACTIONS AND CHEMICAL TRANSPORT OF MOLYBDENUM WITH HALOGENS AND OXYGEN UNDER STEADY STATE CONDITIONS OF INCANDESCENT LAMPS, Mat. res. Bull., 18, pp. 355-369 (1983).

(8) Neumann, G.M: Der mechanismus des wolfram halogenkreisprozesses in halogengluhlampen, LICHTTECHNIK, 24-12, pp. 606-607 (1972).

(9) Eriksson, G.: Thermodynamic Study of High Temperature Equilibria, Acta. Chem. Scand., 25, No7 (1971).

(10) Eriksson, G. and Hack, K. ChemSage-A Computer Program for the Calculation of Complex Chemical Equilibria, Metallurgical transactions, 21B, pp. 10131023 (1990).

(11) Eriksson, G.: AN ALGORITHM FOR THE COMPUTATION OF AQUEOUS MULTICOMPONENT, MULTIPHASE EQUILIBRIA, Analytical Chimica Acta, 112, pp. 375-383 (1979).

(12) Eriksson, G.: Thermodynamic Studies of High Temperature Equilibria, Chemica Scripta, 8, pp. 100103 (1975).

(13) Besmann, T. M: SOLGASMIX-PV, a Computer
Program to Calculate Equilibrium Relationships in Complex chemical Systems, Oak Ridge National Iaboratory TM-5775 (1997).

(14) White, W. B., Johnson S. M. and Dantzig, G. B.: Chemical Equilibrium in Complex Mixtures, Journal of Chemical Physics, 28-5, pp. 751-755 (1958).

(15) Smith, W. R. and Missen, R. W.: Chemical Reaction Equilibrium Analysis, John Wiley \& Sons (1982).

(16) Hack, K: The SGTE casebook, The Institute Materials (1996).

(17) Schnedler, E.: Description of tungsten transport processes in inert gas incandescent lamps, Philips J.Res., 38, p. 224 (1983).

(18) Dittmer, G. and Niemann, U.: THE INFLUENCE OF SOLID PHASE ON TRANSPORT CYCLES IN HALOGEN-FILLED INCANDESCENT LAMPS, Philips J.Res., 42, pp. 41-57 (1987).

(19) Dittmer, G. and Niemann, U.: THERMODYNAMIC CONSIDERATION ON TRANSPORT PROPERTIES OF HALOGEN-FILLED FLOODLIGHT LAMPS, International Symposium on the Science \& Technology of Light Sources (LS5) 22P, pp. 103-104 (1989).

(20) Ooyama, A., Nakagawa, N., Saito, H. and Fuwa, A: Numerical analysis of the thermal and material transport mechanism inside a small incandescent lamp, Journal of the Illuminating Engineering Institute of Japan, 81-2 pp.66-77 (1997) (in Japanese).

(21) Chang, P. Y: Three-dimensional naturalconvection in an A19 100W incandescent lamp, IES Annual Conference Paper 20, pp. 126-139 (1991).

(22) Langmuir, I.: Description of tungsten transportprocesses in incandescent lamps, Phys. Rev., 34, p. 401 (1912).

(23) Ueda, T: Fluid mechanics of reaction systems, Corona Publishing Co. (2002) (in Japanese).

(24) Hirano, T. and Ishizuka, S.: Fluid mechanics, Maruzen (1996) (in Japanese).

(25) Heat Transfer, 4th edition, JSME, p. 356 (1986) (in Japanese).

(26) Rahmane, M. and Meschter, P: Thermodynamics aspect of tungsten chemical transport in high pressure mercury lamps, Proceedings of the 9th international symposium on the science \& technology of light sources, pp. 71-72 (2001). 